\title{
EFECTOS DE LA INCERTIDUMBRE EN LA OPTIMIZACIÓN DE PORTAFOLIOS DE LAS ADMINISTRADORAS DE FONDOS DE PENSIONES COLOMBIANAS PARA 2020
}

The influence of uncertainty on portfolio optimization for Colombian pension funds for 2020

Bibiana Andrea Pardo Castro bpardo89@unisallle.edu.co

Juan Esteban Velasco Mora jvelasc019@unisalle.edu.co

Docente tutor José Rodrigo Vélez Molano jrvelez@unisalle.edu.co

Universidad de La Salle Finanzas y Comercio Internacional

Colombia

\section{RESUMEN}

En el presente estudio se buscó determinar el efecto de la incertidumbre en la estimación del retorno y el riesgo en la conformación de portafolios para las administradoras colombianas de fondos de pensiones obligatorias para el año 2020. Mediante la formulación de tres clases de portafolios de inversión a través de RStudio y la librería CVXR, se procedió a identificar el impacto de la adición de los pasivos pensionales a las participaciones de los activos. Posteriormente, mediante la incorporación de un ruido sobre los parámetros de estimación se compara el efecto de la incertidumbre en las participaciones, para todos los portafolios obtenidos. En el caso del perfil conservador se obtuvo que la adición de los pasivos pensionales no genera un cambio en las participaciones para el portafolio de Markowitz con aversión al riesgo, mientras que en el portafolio de mínima varianza hay variaciones importantes. Adicionalmente, se observó que el efecto de la incertidumbre no genera cambios en las decisiones de inversiones sobre los activos, no obstante, puede llegar a cambiar la proporción de la inversión en un aumento hasta del $40 \%$ para varios escenarios simulados. En síntesis, la investigación hasta el momento logra determinar que es relevante el estudio más profundo de los parámetros de estimación dentro de la optimización realizada por las AFP, además sugiere que se debe considerar la adición de los pasivos pensionales dentro de los portafolios, con el fin gestionar de una mejor forma el riesgo de no pago para los pensionados.

\section{PALABRAS CLAVE:}

Incertidumbre, portafolios, AFP, pasivo pensional, optimización

Cite este artículo como: Pardo, B., \& Velasco, J. (2020). Efecto de la incertidumbre en la optimización de portafolios de las Administradoras de Fondos de Pensiones colombianas para 2020. 


\section{INTRODUCCIÓN}

Las Administradoras de Fondos de Pensiones (AFP en adelante), por su naturaleza como inversionistas a largo plazo, están orientadas a determinar el riesgo de sus inversiones de forma muy distinta a otras entidades, priorizando el balance entre la rentabilidad de cada AFP y las pensiones pagadas en el futuro. Por ende, necesitan emplear medidas que determinen la inversión de manera más estable y favoreciendo la seguridad y la resiliencia del portafolio conformado.

En el ámbito regulativo colombiano, con respecto al sistema pensional, según el analista de la Escuela Nacional Sindical, Héctor Vásquez (2017), el régimen de ahorro individual representa un sistema mayoritariamente conformado por cuentas y depósitos de ahorro. Esto se debe a que, al momento de la jubilación, los pensionados reciben únicamente el capital ahorrado, a pesar de que no sea suficiente. Es importante recalcar que, para la modalidad de renta vitalicia, la pensión está sujeta a retornos sobre inversiones escogidas por la AFP.

Las AFP deben mantener el seguimiento del riesgo de mercado sobre los periodos de incertidumbre financiera, asegurándose de que la estimación en los modelos escogidos para la optimización de portafolios no presente errores estadísticos que puedan perjudicar las obligaciones adquiridas con los aportantes y pensionados en el largo plazo.

De los estudios que evalúan la eficiencia de dichos modelos en Colombia, Jara, Gómez y Amézquita (2005), realizaron una evaluación sobre si las decisiones de estas entidades se encontraban orientadas a portafolios eficientes según el método de optimización de Markowitz (1952), mediante un conjunto de activos seleccionados según la mínima calificación de riesgo y sujetos a las restricciones normativas en la inversión. En el estudio se muestra que en realidad los portafolios de las AFP se encuentran por debajo de la frontera eficiente. Adicionalmente, los autores sugieren que la ineficiencia de los portafolios puede ser ocasionada por el uso de otros tipos de modelos, distintos a Markowitz, dentro de las AFP.

Por otra parte, Martínez, Pava y Peña (2018), determinaron el grado de eficiencia para las AFP en 2018. Para esto realizaron una selección de portafolio, siguiendo la teoría planteada por Markowitz (1952), que se asemeja a la usada en las AFP para cada perfil de riesgo (conservador, moderado y mayor riesgo). Lo anterior, con el fin de construir una frontera eficiente que permitiera comparar el desempeño de los portafolios, sujetos a restricciones estipuladas por el Ministerio de Hacienda y Crédito Público. Finalmente, concluyeron que los portafolios de pensiones se encontraban muy alejados de su frontera eficiente, impidiendo que estos alcanzaran la mayor rentabilidad posible, debido principalmente a los límites relacionados a los activos emitidos por entidades del exterior impuestos por la normativa colombiana.

En el ámbito internacional, Chopra y Ziemba (1993), realizan una serie de experimentos empleando simulaciones aleatorias para detallar la importancia de los errores de estimación en las medias, varianzas y covarianzas por separado. Su estudio prueba que, dentro de la selección de portafolio óptima, los errores en las medias de los retornos tienen un rol vital en el momento de escoger un portafolio balanceado, ya que errores mínimos pueden generar diferencias muy graves entre portafolios, calificando así de manera incorrecta la volatilidad de cada uno de los activos. Además, muestra que los errores en la varianza también pueden generar discrepancias considerables, aunque en menor medida.

Más adelante, Golfarb y lyengar (2003) proponen modelos alternativos robustos que buscan evitar errores de estimación en la conformación de portafolios. Manifiestan que, en la práctica, las optimizaciones como las planteadas por Markowitz (1952) y Sharpe (1964), con frecuencia llegan a ser sensibles ante las perturbaciones en los parámetros del problema, dado que se presentan errores estadísticos en la estimación y resultan poco fiables para la toma de decisiones. También recalcan que, aunque existen técnicas que permiten reducir esta sensibilidad, no tienen garantías sobre el rendimiento del portafolio, de ahí que los modelos robustos consideran que las perturbaciones de los parámetros del mercado son desconocidas y limitadas (conjuntos de 
incertidumbre), y que asumir el peor comportamiento de estas perturbaciones es la mejor manera de resolver los problemas de optimización.

Posteriormente, Sutcliffe y Platanakis (2016) utilizan la optimización robusta dentro del Asset Liability Management (ALM en adelante), para el caso de la AFP USS (Universities Superannuation Scheme), de Reino Unido, usando como medida de desempeño el Ratio de Sharpe. No obstante, sustentan su análisis en la posibilidad de que haya errores de estimación en los parámetros del modelo. Por lo tanto, transforman la optimización al aplicar conjuntos de incertidumbre en la media y la varianza, en orden de identificar y eliminar las soluciones dadas por posibles errores en la estimación. Esta selección de los activos del portafolio de inversión se realiza teniendo en cuenta aquellos permitidos por la regulación del Reino Unido, y aquellos más usados por la USS dentro de los límites de inversión establecidos legalmente. Los autores determinaron que, dentro de los cuatro modelos empleados, el modelo de optimización robusta obtuvo el mejor desempeño histórico, además de que este modelo presenta el portafolio con mayor estabilidad a lo largo del tiempo.

Simultáneamente, Duarte, Valladão y Veiga (2017), desarrollan una optimización mediante programación estocástica. En esta investigación se observa el desempeño de las AFP brasileñas dentro de múltiples escenarios simulados en una distribución de probabilidad uniforme, los cambios están sujetos a restricciones sobre el capital mínimo y los indicadores de insolvencia dados por los requerimientos del Solvencia II, junto a diferentes escenarios en los pasivos de las AFP según la probabilidad de muerte de los aportantes y pensionados. Esta metodología requiere de una gran carga computacional al momento de programar y ejecutar el modelo, además de plantear penalidades monetarias a las AFP en caso de incumplir las restricciones, aspecto que en un contexto práctico es poco común. Este estudio concluyó que las restricciones definidas por la regulación de Brasil contribuyen a incrementar la aversión al riesgo de la compañía a lo largo del tiempo; este efecto se puede evidenciar ya que, si las restricciones del modelo se vuelven laxas, se genera un aumento en la probabilidad de insolvencia.

Debido a los problemas en la selección de portafolio, en las teorías y modelos mencionados anteriormente, la presente investigación permitirá comprender y explorar en qué forma la incertidumbre aplicada a los parámetros de estimación afecta las participaciones de los activos y pasivos en los portafolios de las AFP en Colombia. La manera en que este estudio contribuirá al análisis pensional se puede explicar en dos componentes, la adición de pasivos pensionales a los portafolios de activos conformados por las AFP, y posteriormente, la comparación entre una optimización de portafolios con y sin incertidumbre.

En el periodo de análisis escogido también resaltan dos grandes eventos en el ejercicio de ALM y la conformación de portafolios de los fondos de pensiones. En el año 2019, las AFP registraron el mejor rendimiento hasta la fecha, reportando $\$ 40$ billones para los afiliados. Por otra parte, el primer semestre de 2020 se caracterizó, dada la coyuntura económica ocasionada por la pandemia, por rendimientos negativos para las AFP. Dichos cambios sugieren el uso de nuevas metodologías que brindarán una perspectiva más amplia sobre la variabilidad en los portafolios a las que se está expuesta una entidad en periodos de estrés.

\section{MÉTODO}

Esta investigación tiene un enfoque cuantitativo con alcance descriptivo, ya que según Sampieri (2014), se busca realizar un estudio sobre variables cuantificables, como lo son los rendimientos y precios de activos financieros, con el fin de probar las hipótesis planteadas anteriormente. El estudio parte de una revisión teórica sobre la estructuración, funcionamiento y aplicación práctica de la optimización de portafolios, por lo tanto, necesita un tratamiento estadístico en un determinado contexto, en donde se deben medir las variables mediante instrumentos de estimación, en orden de determinar sus características principales. 


\section{Recolección de datos}

La recolección de datos para la presente investigación se realizará por medio de datos secundarios. Para los activos se tendrá en cuenta sus categorías, por esta razón se tomará como referente los once seleccionados en el trabajo de Martínez et al. (2018), que son:

\begin{tabular}{|c|c|}
\hline Activos & Categoría \\
\hline iColcap & Acciones de Colombia \\
\hline iShares Europe ETF (IEV) & Acciones de Europa Continental - Rendimiento \\
\hline iShares JPMorgan USD Emerging Markets Bond (EMB) & Bonos de deuda pública de países emergentes \\
\hline iShares JPX- Nikkei 400 ETF (JPXN) & Acciones de Japón \\
\hline Barclays International Trs Bd ETF (BWX) & Bonos de deuda pública países desarrollados \\
\hline Vanguard FTSE Europe ETF (VGK) & Acciones de Europa Continental - Capitalización \\
\hline Fonval Global Renta Fija (FONVALGB) & Fondo colombiano de inversión internacional \\
\hline Renta Liquidez Valores Bancolombia (CARENTLIUD) & Fondo colombiano de inversión nacional \\
\hline COLTES & Bonos de deuda pública de Colombia - Nominal \\
\hline COLTES UVR & Bonos de deuda pública de Colombia - Real \\
\hline Standard \& Poor's 500 (S\&P500) & Acciones de Estados Unidos \\
\hline
\end{tabular}

Posteriormente, se consultarán las bases de datos Yahoo Finance, Bolsa de Valores de Colombia y la Superintendencia Financiera para descargar la información mensual en el periodo establecido.

Sutcliffe et al. (2016) definen tres clases de pasivos pensionales, con base en esto se realizará una transformación que permitirá adaptarlos al contexto colombiano, por medio de las definiciones dadas en la Resolución 1056 de 2015 del Ministerio de Salud y Protección Social. De esta manera, los pasivos serán:

\begin{tabular}{|c|c|}
\hline Pasivos aplicados por Sutcliffe et al. (2016) & Pasivos equivalentes para Colombia \\
\hline Miembros activos & Miembros activos cotizantes \\
\hline Miembros pensionados & Miembros pensionados \\
\hline Miembros diferidos & Miembros activos no cotizantes \\
\hline
\end{tabular}

En orden de calcular el valor presente de los pasivos pensionales, es necesario la recopilación de una extensa cantidad de variables para un solo periodo, las cuales también son utilizadas como variables intermedias dentro de la formulación final planteada en el trabajo de Sutcliffe et al. (2016). Las variables serán recolectadas exclusivamente para el periodo entre enero de 2019 hasta junio de 2020, en coherencia con los eventos relevantes dentro de la gestión de las AFP colombianas planteadas en la justificación. Además, como se emplearán algunas variables pronosticadas, se considera que un periodo de tiempo más extenso implicaría perder la relevancia de los pronósticos actuales. A continuación, se presentará una síntesis de la información correspondiente a las bases de datos consultadas para las variables, junto con el tipo de pasivo seleccionado que las requiere.

\begin{tabular}{|c|c|c|c|c|}
\hline Variables & Bases de datos utilizadas & Activos Cotizantes & $\begin{array}{l}\text { po de pasivo seleccionad } \\
\text { Miembros } \\
\text { Activos No Cotizantes }\end{array}$ & Pensionados \\
\hline Salario promedio & Banco de la República & $\mathrm{x}$ & $x$ & \\
\hline Edad de retiro & $\begin{array}{l}\text { Artículo } 9 \text { de la } 797 \text { de } \\
2003 \text { que modifica } \\
\text { artículo } 33 \text { de la Ley } 100 \\
\text { de } 1993\end{array}$ & $\mathrm{x}$ & $\mathrm{x}$ & $\mathrm{X}$ \\
\hline Esperanza de vida & Acosta \& Romero (2014) & $\mathrm{x}$ & $\mathbf{x}$ & $\mathrm{x}$ \\
\hline Pensión promedio & $\begin{array}{l}\text { Fundación para la } \\
\text { Educación Superior y el } \\
\text { Desarrollo }\end{array}$ & & & $\mathrm{X}$ \\
\hline
\end{tabular}




\begin{tabular}{|c|c|c|c|c|}
\hline Tasa de descuento & $\begin{array}{ll}\text { Cálculo Curva } & \text { de } \\
\text { Rentabilidad Tasa } & \text { Fija } \\
\text { TES del Grupo Aval } & \end{array}$ & $x$ & $x$ & $x$ \\
\hline $\begin{array}{l}\text { Tasa de crecimiento del } \\
\text { salario pronosticada }\end{array}$ & Banco de la República & $\mathrm{x}$ & & \\
\hline $\begin{array}{l}\text { Tasa de inflación } \\
\text { pronosticada }\end{array}$ & Banco de la República & $x$ & $\mathrm{x}$ & $\mathrm{x}$ \\
\hline $\begin{array}{l}\text { Tasa de colocación del } \\
\text { fondo } \\
\text { Variables ponderadas }\end{array}$ & $\begin{array}{l}\text { Metodología de Sutcliffe } \\
\text { et al. } \\
\text { Bases de datos utilizadas }\end{array}$ & $x$ & $x$ & \\
\hline Edad promedio & $\begin{array}{l}\text { Superintendencia } \\
\text { Financiera }\end{array}$ & $x$ & $x$ & \\
\hline $\begin{array}{l}\text { Años promedio de } \\
\text { aporte }\end{array}$ & $\begin{array}{l}\text { Superintendencia } \\
\text { Financiera }\end{array}$ & $x$ & $x$ & \\
\hline Número de miembros & $\begin{array}{l}\text { Superintendencia } \\
\text { Financiera }\end{array}$ & $x$ & $x$ & $x$ \\
\hline
\end{tabular}

Tabla 3. Bases de datos utilizadas para el cálculo de los pasivos pensionales seleccionados. Elaboración propia

Fases

Para el estudio de los datos se tomará como guía el trabajo de Palomar (2019), con respecto a las técnicas de optimización de portafolios con incertidumbre, mediante el uso del software estadístico R Studio con el paquete CVXR. Desde aquí, la metodología para el análisis y la interpretación de la información seguirá cuatro fases de desarrollo: la primera fase corresponde al tratamiento previo de los pasivos; en cuanto a la segunda, tercera y cuarta fase hacen referencia a los objetivos específicos de la investigación.

Es importante mencionar que el problema se desarrollará mediante el planteamiento hipotético de una administradora que refleje el comportamiento promedio de las AFP vigiladas por la Superintendencia Financiera actualmente. Por lo tanto, en la primera fase se realizarán promedios ponderados entre las AFP, para estimar las variables ponderadas mencionadas en la Tabla 3 según cada perfil de riesgo. Una vez hecho el tratamiento de las variables, se procederá a estimar el valor pensional de cada tipo de obligación del fondo, según la metodología expresada en el Apéndice D de Sutcliffe et al. (2016), en orden de hallar las proporciones asignadas para la posterior conformación del portafolio.

En la segunda fase se planteará la optimización de los portafolios de mínima varianza, máximo retorno y Markowitz con aversión al riesgo, para cada uno de los perfiles de riesgo. Inicialmente, los portafolios estarán conformados a partir de una variación porcentual de los activos, con restricciones que no permiten operaciones en corto, ni invertir más allá del capital del fondo, así como restricciones a la inversión establecidas en el Decreto 2555 de 2010. Se establecerán nuevas restricciones para los tres pasivos, en donde las proporciones permanecen fijas y negativas, en orden de contrarrestar la inversión total en activos y equilibrar el balance del fondo. Lo anterior, para determinar la diferencia en las participaciones de los activos, una vez incluidos los pasivos.

Posteriormente, en la tercera fase se aplicará incertidumbre mediante una generación de números aleatorios distribuidos normalmente, en orden de aplicar ruido a la estimación de la media y/o la varianza de los retornos, según corresponda al tipo de portafolio. Este proceso se realizará por mil iteraciones y se recopilará la información sobre la frecuencia en la que varían las participaciones, con el fin de obtener una muestra de datos suficientes que den certeza sobre el efecto de la incertidumbre.

Por último, partiendo de las estimaciones obtenidas de los portafolios con y sin incertidumbre, en la cuarta fase se construirán histogramas de frecuencias para cada uno de los once activos seleccionados. Esto con el fin de comparar la intensidad de los cambios en las participaciones, con respecto a aquellas obtenidas con los parámetros de estimación de cada portafolio original. Adicionalmente, se observarán las variaciones en las participaciones de los activos entre los perfiles de riesgo, según cada portafolio. 


\section{RESULTADOS}

A continuación, se presentan los resultados preliminares obtenidos hasta el momento, es decir, las cuatro fases de la metodología para el perfil conservador.

Para tal fin, primero se estimó el portafolio de Mínima Varianza para la AFP, conformada como se presenta en las imágenes 1 y 2; se observó que las participaciones se encuentran distribuidas mayoritariamente en cuatro activos, los cuales son FONVALGB (A7), CARENTLIUD (A8), COLTES UVR (A10) y S\&P500 (A11), esto puede explicarse a que la varianza de los activos 7 Y 8 son las menores por un amplio margen. No obstante, cuando se incluyen las proporciones del pasivo pensional correspondientes al perfil conservador, se evidencia que gran parte de los recursos destinados al activo 7 y al activo 10 se redirigen al activo 5 (BWX), esto implica que al momento de tomar decisiones de inversión para equilibrar el desbalance ocasionado por el pasivo se tiene como criterio aspectos diferentes a una menor volatilidad individual.

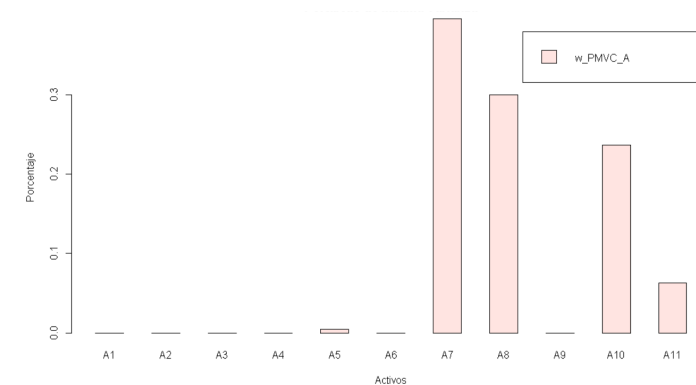

Imagen 1. Portafolio de Mínima Varianza solo activos. Elaboración propia

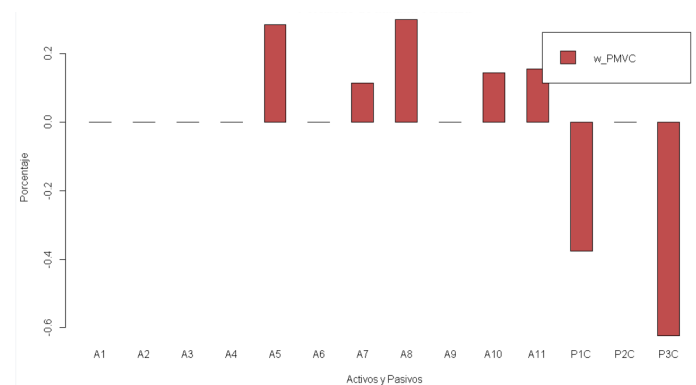

Imagen 2. Portafolio de Mínima Varianza activos y pasivos. Elaboración propia

Segundo, en las imágenes 3 y 4 las participaciones de los activos no cambiaron para la estimación del portafolio de Markowitz con aversión al riesgo, a pesar de la influencia de los pasivos pensionales, manteniéndose la inversión principalmente entre el activo 9 (COLTES) y el activo 11.

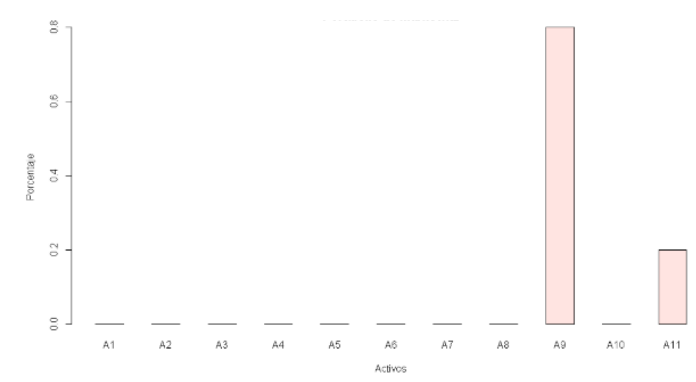

Imagen 3. Portafolio Markowitz solo activos. Elaboración propia

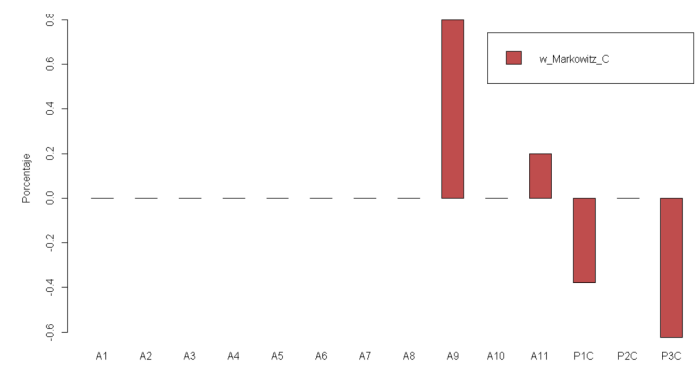

Imagen 4. Portafolio de Markowitz activos y pasivos. Elaboración propia

Una vez establecidos los portafolios principales, se procedió a cuestionar la confiabilidad del parámetro de estimación mediante la generación iterativa de valores aleatorios que a su vez hacen de retornos, distribuidos con la media y la varianza de los retornos de los activos. La información sobre la participación de cada activo en la optimización de portafolios en todas las simulaciones se sintetizó dentro de histogramas de frecuencia. 


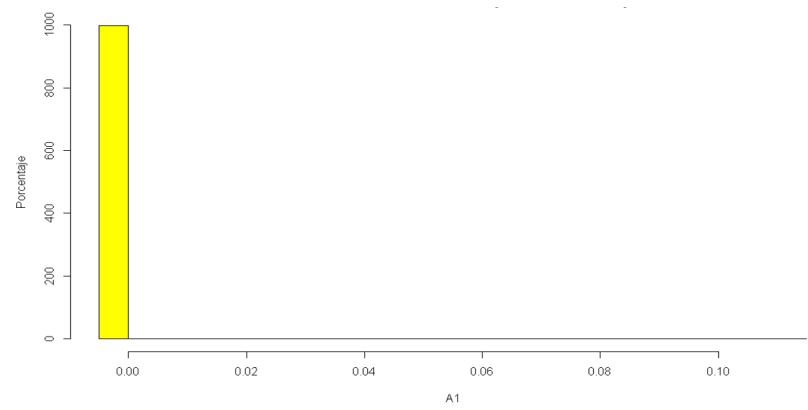

Imagen 5. Portafolio de Mínima Varianza activo 1. Elaboración propia

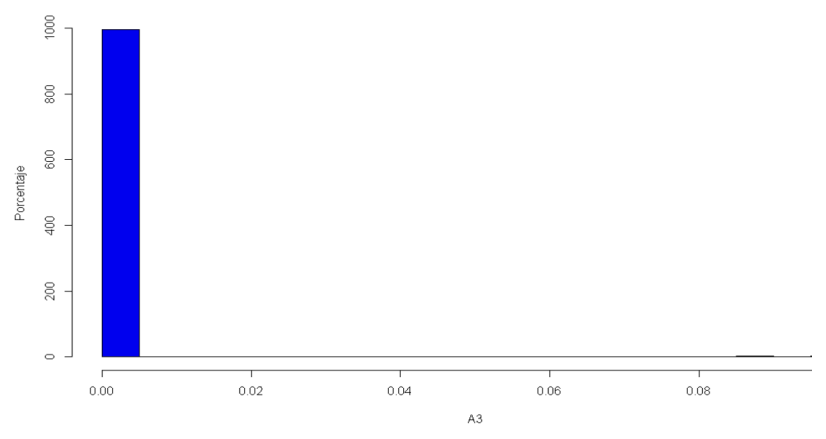

Imagen 7. Portafolio de Mínima Varianza activo 3. Elaboración propia

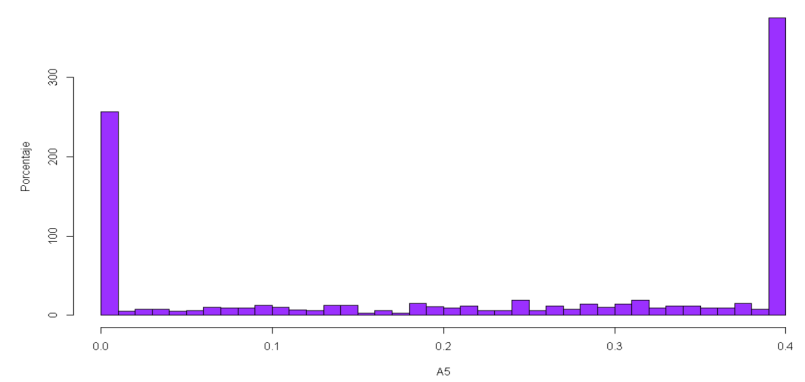

Imagen 9. Portafolio de Mínima Varianza activo 5. Elaboración propia

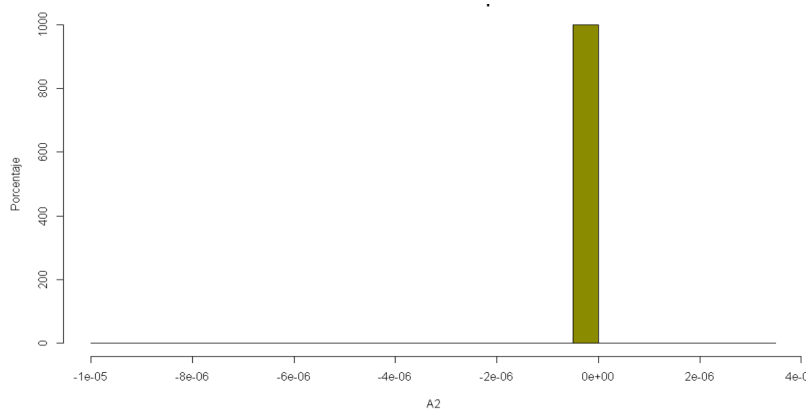

Imagen 6. Portafolio de Mínima Varianza activo2. Elaboración propia

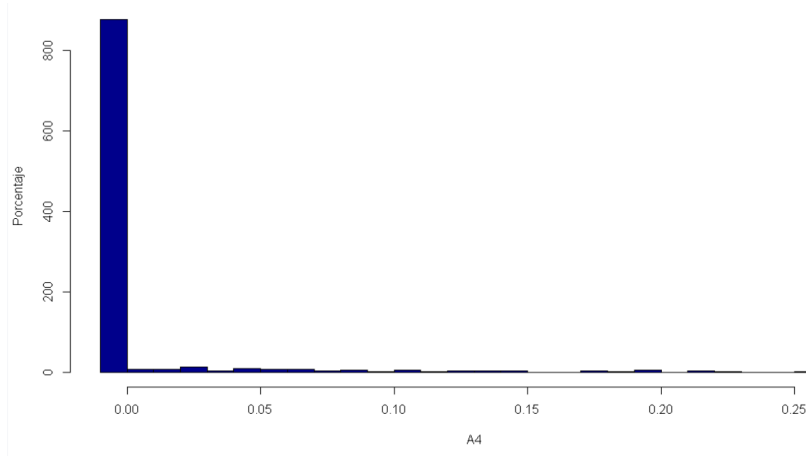

Imagen 8. Portafolio de Mínima Varianza activo 4. Elaboración propia

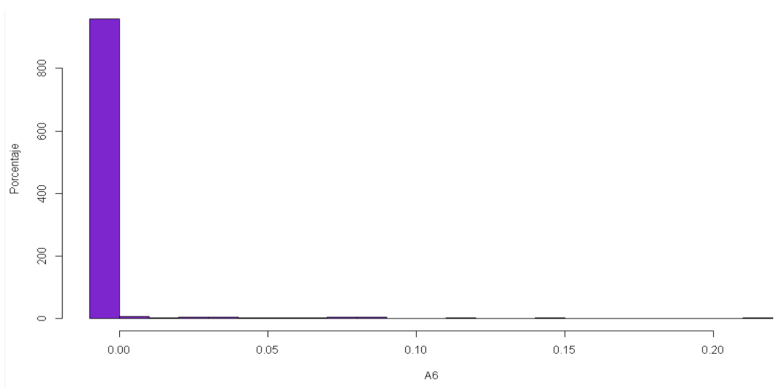

Imagen 10. Portafolio de Mínima Varianza activo 6. Elaboración propia
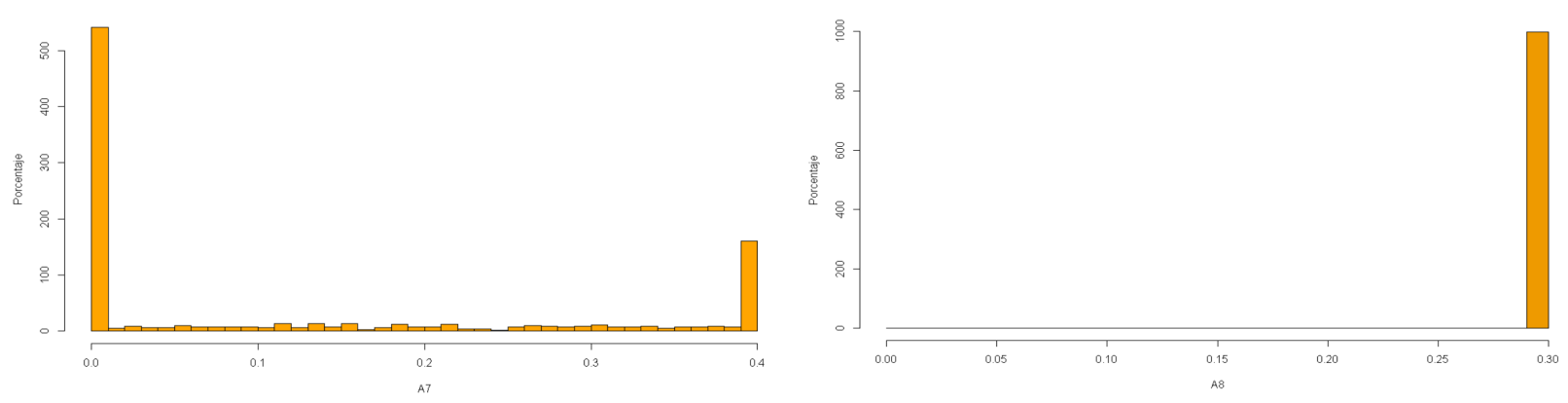
Imagen 11. Portafolio de Mínima Varianza activo 7. Elaboración propia

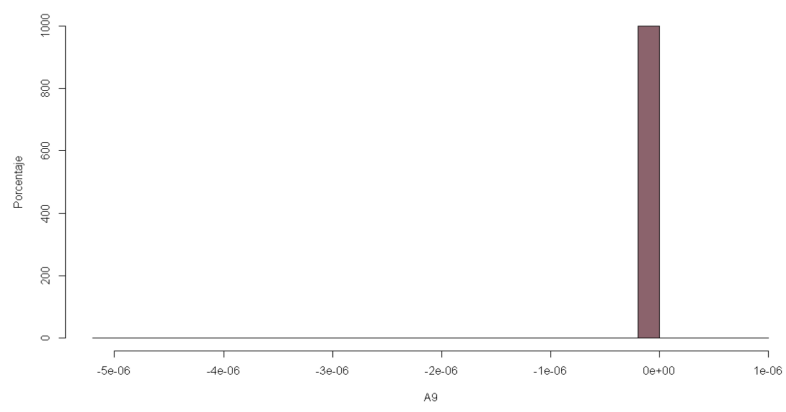

Imagen 13. Portafolio de Mínima Varianza activo 9. Elaboración propia
Imagen 12. Portafolio de Mínima Varianza activo 8. Elaboración propia

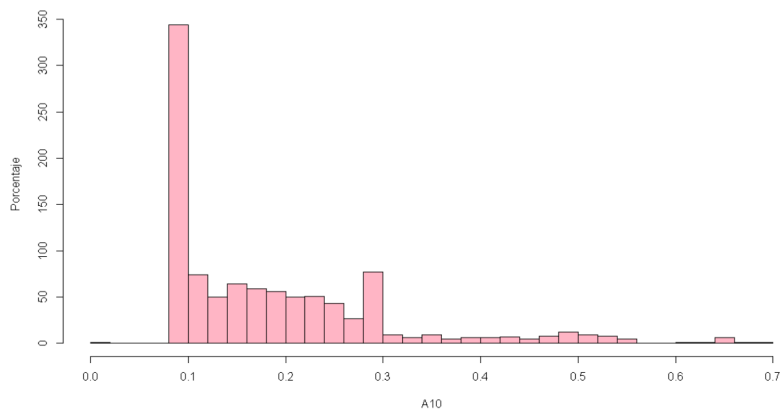

Imagen 14. Portafolio de Mínima Varianza activo 10. Elaboración propia

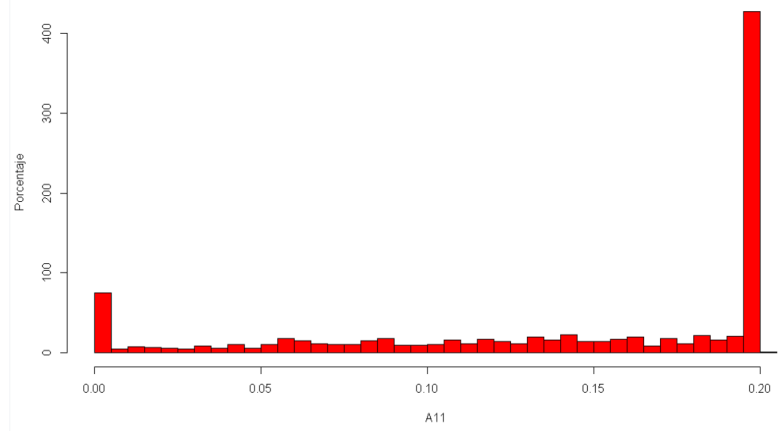

Imagen 15. Portafolio de Mínima Varianza activo 11. Elaboración propia

Dentro del portafolio de Mínima Varianza se observa que los activos que están más expuestos a cambios en sus participaciones por consecuencia de errores en la estimación son el activo 5, 7, 10 y 11. Lo anterior muestra que el efecto de la incertidumbre sobre el portafolio no es suficiente para cambiar la selección de activos, pero sí sus participaciones. Para el activo 5 se puede observar que la cuarta parte de las simulaciones obtenidas no le otorgan una participación relevante, sin embargo, para la tercera parte de estas simulaciones su participación es del 40\%. El activo 7 presenta un comportamiento similar al del activo 5, pero más de la mitad de las simulaciones se concentra en un nulo, y cerca de 200 de las simulaciones están en un 40\%. En el caso del activo 10 sus participaciones son mucho más variadas, encontrándose entre un 10\% y un 30\% para grupos de más de 50 simulaciones, no obstante, la mayor participación es del 10\% para cerca de 350 simulaciones. Igualmente, para el activo 11 en más de 400 simulaciones obtiene una participación de $20 \%$, para 80 simulaciones, un $0 \%$ y existen grupos de menos de 20 simulaciones que obtienen participaciones que se distribuyen entre el $1 \%$ y el $19 \%$.

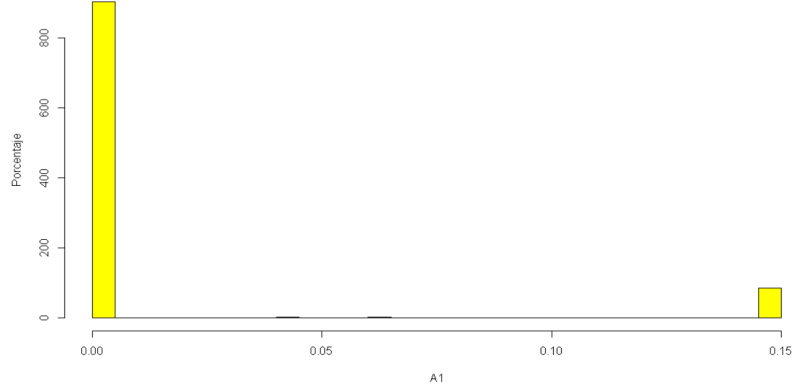

Imagen 16. Portafolio de Markowitz activo 1. Elaboración propia

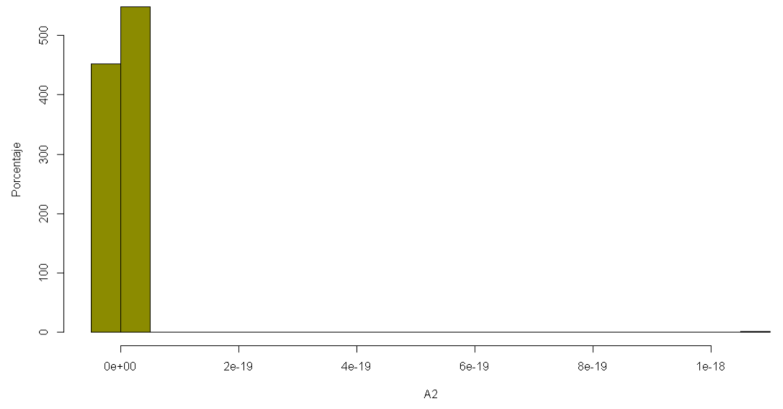

Imagen 17. Portafolio de Markowitz activo2. Elaboración propia 


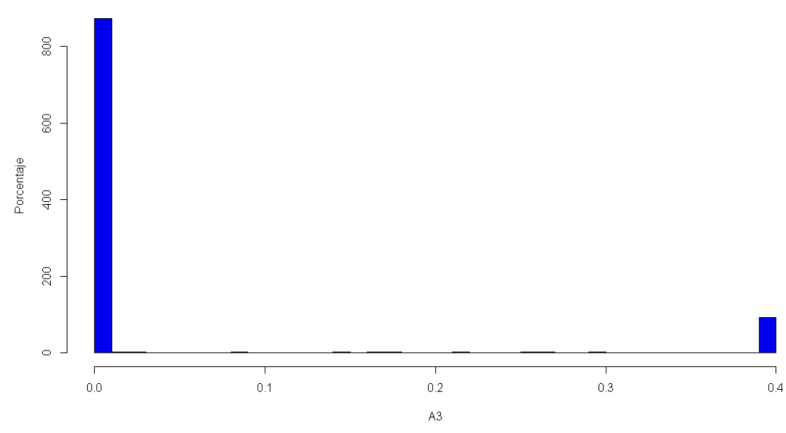

Imagen 18. Portafolio de Markowitz activo 3. Elaboración propia

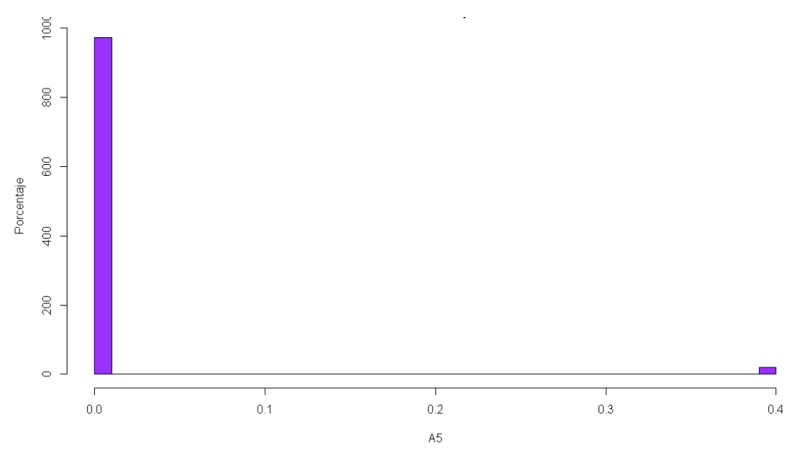

Imagen 20. Portafolio de Markowitz activo 5. Elaboración propia

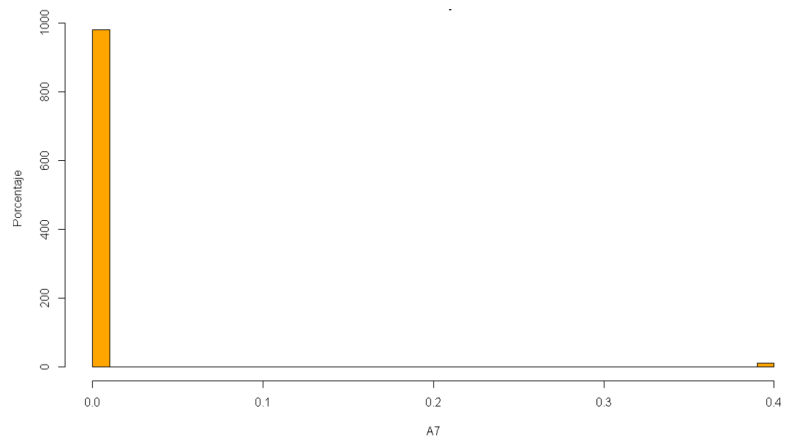

Imagen 22. Portafolio de Markowitz activo 7. Elaboración propia

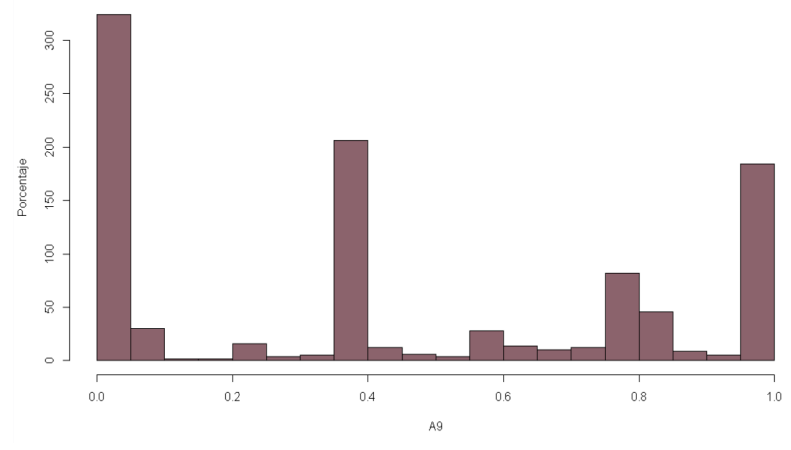

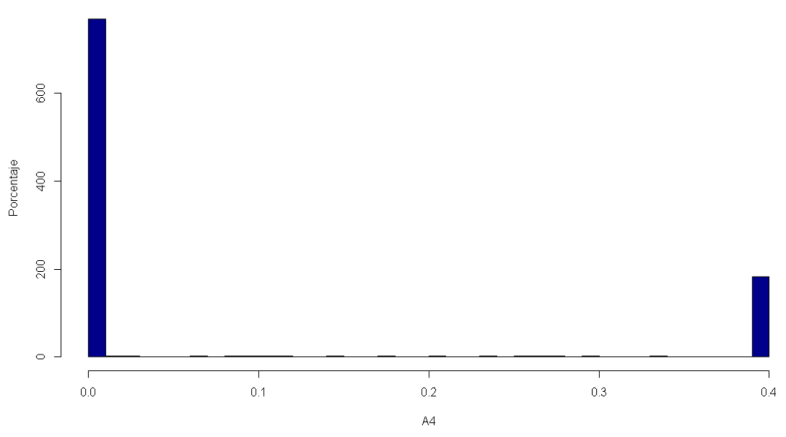

Imagen 19. Portafolio de Markowitz activo 4. Elaboración propia

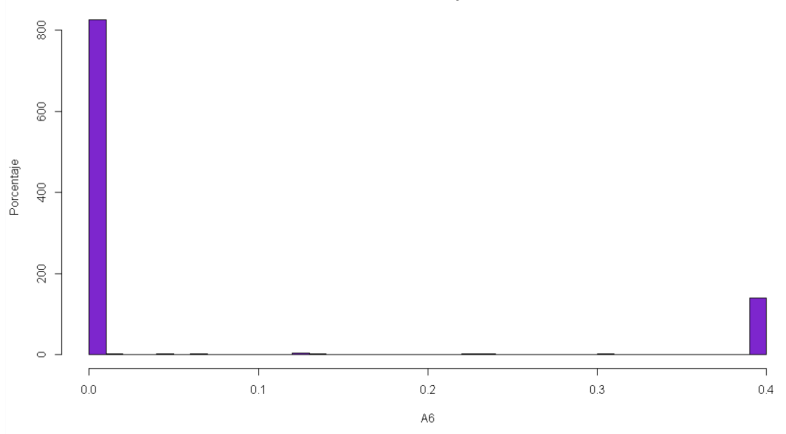

Imagen 21. Portafolio de Markowitz activo 6. Elaboración propia

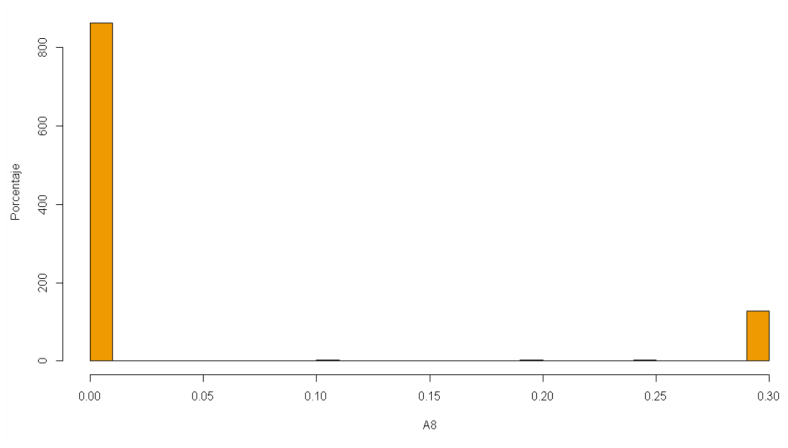

Imagen 23. Portafolio de Markowitz activo 8. Elaboración propia

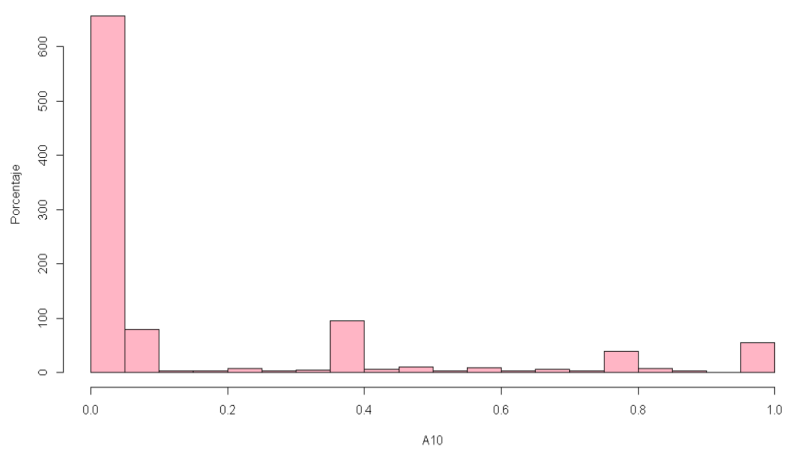




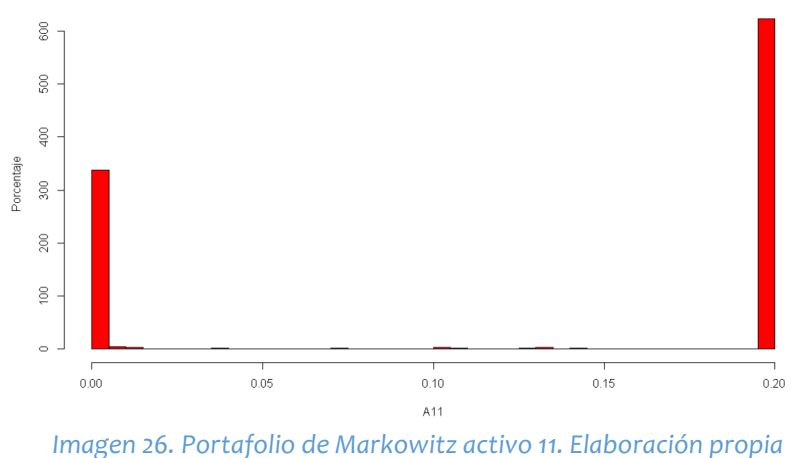

Por otra parte, en el portafolio de Markowitz con aversión al riesgo se evidencia que los activos que están más expuestos a las variaciones en sus participaciones son el activo 9,10 y 11. Al igual que con el portafolio de Mínima Varianza, el efecto de la incertidumbre no alcanza a cambiar la selección de activos de manera drástica, aunque cabe destacar que el activo 10 es la excepción. En el activo 9 existe una variación extrema que no presenta patrones definidos, ya que se observa que las mayores participaciones son del $0 \%$ para 400 simulaciones y menos de 200 simulaciones para el 40\% y 100\%. Para el activo 10 las participaciones varían entre $10 \%, 40 \%, 80 \%$ y $100 \%$ para grupos de menos de 100 simulaciones, no obstante, la mayor cantidad de observaciones tiene una participación nula. Finalmente, para el activo 11 sus participaciones principalmente se distribuyen en el $0 \%$ y $20 \%$ con más de 300 y 600 simulaciones respectivamente.

\section{DISCUSIÓN Y CONCLUSIÓN}

De los resultados preliminares, se puede observar que la adición de los pasivos pensionales a la optimización de portafolios puede llegar a afectar de manera considerable las participaciones de los activos. Esto se debe probablemente a que dentro de la optimización con pasivos el riesgo adicional dado por las obligaciones aumenta el riesgo general del portafolio, y esto lleva a que, dentro del cálculo de las participaciones, se busque otros activos, que originalmente eran más riesgosos, para lograr compensar este riesgo.

El análisis orientado a la medición de incertidumbre a partir de este trabajo hasta el momento logra determinar que es relevante el estudio más profundo de los parámetros de estimación, puesto que en los resultados preliminares se observa la sensibilidad implícita que poseen la media y la varianza ante comportamientos atípicos que puedan generar cálculos erróneos sobre la muestra de los datos.

Sin embargo, en la presente investigación se encontró que el cálculo actuarial del pasivo pensional es un proceso que consume mucho tiempo y requiere mucha información. Por esta razón, para la adopción del pasivo al análisis del portafolio se propone realizar una fase previa de estudios particulares de las variables que afectan a los pasivos en el largo plazo. No obstante, el cálculo del pasivo pensional no tiene en cuentas las variaciones en el tiempo, por lo tanto, un enfoque estocástico para los pasivos pensionales resultaría más apropiado, aunque la dificultad para la estimación del modelo incrementaría de forma drástica, principalmente por la extrema cantidad de escenarios posibles a calcular que este requiere en el largo plazo.

Finalmente, se recomienda la aplicación de este tipo de modelos en otro tipo de entidades orientadas a la gestión de obligaciones en el largo plazo para el caso colombiano, como las compañías de seguros. También se sugiere la profundización en los modelos que tienen en cuenta la incertidumbre, como los modelos de optimización robusta y programación estocástica, al igual que el uso de otras metodologías a comparar distintas al modelo de Markowitz. 


\section{REFERENCIAS BIBLIOGRÁFICAS}

Acosta, K., \& Julio, R. (2014). Cambio de las recientes causas de mortalidad en Colombia. Documentos de Trabajo Sobre Economía Regional, Banco de La República - Centro de Estudios Económicos Regionales (209), 141. Recuperado

de: https://www.banrep.gov.co/sites/default/files/publicaciones/archivos/dtser_209.pdf

Amaya, J. (2020). Los fondos de pensiones privados evidenciaron pérdidas en rendimientos al primer trimestre de 2020, pero aseguran que desde abril se ven recuperaciones. La República. Recuperado de: https://www.larepublica.co/finanzas/las-razones-por-las-que-cayo-el-ahorro-pensional-a-marzo-yextractos-reflejan-bajas-2999072

Chopra, V., \& Ziemba, W. (1993). The effect of errors in means, variances and covariances on optimal portfolio choice. Journal of Portfolio Management, 611 Recuperado de: https://faculty.fuqua.duke.edu/ _charvey/Teaching/BA453_2006/Chopra_The_effect of $1993 . p d f$

Daza-Orozco, CE. (2018). Notas acerca de la investigación en administración de empresas. Revista Fusión. Bogotá. Fundación Universitaria San Mateo. Retrieved from http://cipres.sanmateo.edu.co/index.php/fusion/article/view/38

Dinero. (2020) ¿Busca pensionarse de un fondo privado? Noticia de $\$ 40$ billones. Recuperado de: https://www.dinero.com/inversionistas/articulo/resultados-fondos-de-pensiones-en-2019/282065

Duarte, T., Valladão, D., \& Veiga, Á. (2017). Asset liability management for open pension schemes using multistage stochastic programming under Solvency-II-based regulatory constraints. Insurance: Mathematics and Economics, $\quad 77, \quad$ 177-188. Recuperado de: https://www-sciencedirectcom.hemeroteca.lasalle.edu.co/science/article/pii/S0167668717302081?via\%3Dihub

Goldfarb, D., \& lyengar, G. (2003). Robust portfolio selection problems. Mathematics of operations research, 28(1), 1-38. Recuperado de: https://www.jstor.org/stable/4126989

Hernández, R., Fernández, C., \& Baptista, P. (2014). Metodología de la investigación. 6ta Edición McGraw-Hill

Jara, D., Gómez, C., \& Amézquita, A. (2005). Análisis de la eficiencia de portafolios pensionales obligatorios en Colombia. Revista ESPE (49), 192-239. Recuperado de: https://www.banrep.gov.co/sites/default/files/eventos/archivos/JaraAFPs2005_0.pdf

Lubinska, B. (2018). Contemporary Challenges for The Asset-Liability Managment. Research Papers of the Wroclaw University of Economics (519), 135-145. Recuperado de:

Markowitz, H. (1959). Portfolio Selection, efficient diversification of investments. Cowles Foundation for Research in Economics at University. Recuperado de: http://cowles.yale.edu/sites/default/files/files/pub/mon/m16-all.pdf

Martínez, N., Pava, V., \& Peña, K. (2018). Evaluación de la eficiencia de los portafolios de los Fondos de Pensiones privados en Colombia para 2018. (Tesis de pregrado). Universidad de la Salle, Bogotá, Colombia.

Mora-Ramírez, Á. J., \& Norman-Acevedo, E. (2018). A systematic review of the literature on the internationalization of the SME enterprise in Colombia. Espacios, 39(3).

Mora-Ramírez, A. J., \& Norman Acevedo, E. (2017). Revisión sistemática de literatura sobre la internacionalización de la empresa PYME en Colombia. Espacios, 38(39).

Ministerio de Hacienda y Crédito Público. (2010). Libro 6 [Entidades Sujetas a inspección y vigilancia y sujetas a control]. Por el cual se recogen y reexpiden las normas en materia del sector financiero, asegurador y del mercado de valores y se dictan otras disposiciones. [Decreto 2555 de 2010]. DO: 47.771

Ministerio de Salud y Protección Social. (2015). Sección 2 del Anexo técnico 1. [Glosario de campos del anexo técnico 1]. Por la cual establecen los anexos técnicos de Registro Único de Afiliados (RUAF), el mecanismo de transferencia de los archivos y se dictan otras disposiciones. [Resolución 1056 de 2015]. DO: 49.478.

Palomar, D. (2019) Robust Portfolio Optimization. [Diapositivas]. Home page of Daniel Palomar. Recuperado de: https://palomar.home.ece.ust.hk/MAFS6010R_lectures/slides_robust_portfolio.html\#

Sharpe, W. (1964). Capital asset prices: A theory of market equilibrium under conditions of risk. The journal of finance, 19(3), 425-442. Recuperado de: https://www.jstor.org/stable/2977928 
Superintendencia Financiera. (1995). Circular Básica Contable Y Financiera. [Circular Externa No.100 de 1995]. Recuperado de: https://www.superfinanciera.gov.co/inicio/normativa/normativa-general/circularbasica-contable-y-financiera-circular-externa--de---15466

Sutcliffe, C., \& Platanakis, E. (2016). Asset Liability Modelling and Pension Schemes: The Application of Robust Optimization to USS. SSRN Electronic Journal, 1-40.

Uribe Arévalo, A., \& Norman Acevedo, E. (2020). La internacionalización de la pequeña y mediana industria del software y de las tecnologías informáticas (SW \&amp; TI) a través del efecto trampolín del gremio. Cuadernos Latinoamericanos De Administración, 16(31). https://doi.org/10.18270/cuaderlam.v16i31.3068

Vásquez, H. (2017). Necesitamos un sistema pensional universal, no sistema de ahorros. Agencia Laboral de Información. Recuperado de: https://ail.ens.org.co/opinion/necesitamos-sistema-pensional-universalno-sistema-ahorros-opinion/ 